\title{
Emotion meets action: Towards an integration of research and theory
}

\author{
Bernhard Hommel $^{1}$, Agnes Moors ${ }^{2}$, David Sander ${ }^{3} \&$ Julien Deonna ${ }^{4}$ \\ ${ }^{1}$ Leiden University \\ ${ }^{2}$ KU Leuven, Ghent University \\ ${ }^{3}$ Swiss Center for Affective Sciences
}

Corresponding Address:

Bernhard Hommel

Institute for Psychological Research \& Leiden Institute for Brain and Cognition Wassenaarseweg 52

2333 AK Leiden, The Netherlands

hommel@fsw.leidenuniv.nl 
Emotion and action have been suspected to be strongly interrelated since the beginning of modern theorizing on emotion. For instance, Darwin (1872) captured his main idea about the evolutionary function of emotions in his principle of serviceable associated habits, according to which the bodily expression of emotions helps to regulate the processing of the emotion-inducing stimulus event. Indeed, recent research has demonstrated that the facial expression of disgust minimizes the sensory processing of information from the disgusting source, while the opposite holds for the facial expression of fear (Susskind et al., 2008). James (1884) put stronger emphasis on the interactions between emotion and action in the phenomenal experience of emotions. According to his approach, it is through perceiving our bodily response to an affective event that, when this response is sufficiently intense, we consciously experience a particular emotion. Indeed, numerous findings indicate that having people move in particular ways (e.g., activate facial muscles involved in smiling or pushing objects away from their body) tends to modulate the experience of the emotion associated with these movements (e.g., Niedenthal, Barsalou, Winkielman, Krauth-Gruber, \& Ric, 2005). A large part of the research on emotion in the 20th century has focused on expressions of emotion, in particular facial expressions. This of course corresponds to an important link between emotion and action, but the mechanisms underlying the production of such expressions is still highly debated (e.g., affect programs vs. appraisals). When it comes to action, more generally than expressions, these insights into the interwoven character of emotion and action have been lost out of sight in many emotion theories and most action theories from the 20th century, however. In action theories, emotions are often reduced to feelings, while action is mainly seen as being driven by particular stimulus conditions. Emotion theories, in turn, tend to give some role to action tendencies - be it as a precondition or as a consequence of feelings_- but the mechanisms underlying these relations are still 
poorly understood. Today, the psychology, neuroscience, and philosophy of action have rediscovered the role of emotion or feelings for action and action control, while the psychology, neuroscience, and philosophy of emotion have rediscovered the embodiment of people's emotions in and through their actions. This provides new opportunities for integrating emotion and action both theoretically/conceptually and empirically/experimentally. The purpose of this special section is to bring together leading theorists and experimental researchers in the areas of action control and emotion, with the ambition to sketch avenues towards such an integration. The contributions and the discussions leading to the special section emerged from a dedicated expert colloquium held in Geneva, organized by Moors, Deonna, Sander, and Hommel, which goes back to ideas and stimulating suggestions from Wolfgang Prinz and Klaus Scherer.

\section{Contributions}

The five target articles in this special section were selected to shine their light on central issues in the psychology, neuroscience, and philosophy concerning the relationship between emotion and action. As the reader will see, there is considerable overlap between these articles, some disagreement and, unsurprisingly with only five contributions, some neglect of other issues that would just as well have deserved consideration (see next section).

Scarantino (2017) discusses and criticizes two arguments against the possibility that emotions can cause actions. For one, emotions have been argued to emerge from, and thus come after action (James, 1884), which would suggest the opposite causal sequence. Scarantino objects that James's view relies on a reduction of emotions to feelings and posits that some feelings may well arise after actions have begun. If emotions are equated with affect programs (Scarantino, 2017), action tendencies (Frijda, 2007), or a set of components that excludes actions (Scherer, 2009), however, there is no reason to give up the common- 
sense view that emotions cause actions. For another, the observation that emotions are not necessarily followed by actions has been taken to rule out that emotions cause actions. Here, Scarantino objects that it would be too naïve to assume a direct, one-to-one relationship between emotions and action. Rather than mechanistically triggering an action, an emotion should be assumed to raise the probability of a particular action.

Blakemore and Vuilleumier (2017) point out that the connection between emotion and cognition has received much more attention in research and theorizing than the link between emotion and action. Recent behavioral and neuroscientific research suggests that the latter link is rather close. Affective events have been shown to systematically trigger corresponding action tendencies, such as approach tendencies to positive and avoidance tendencies to negative events, and to systematically bias actioncontrol processes, such as response inhibition. Echoing Scarantino's argument on this issue (2017), they claim that, rather than being primitive and hard-wired, these response tendencies are sensitive to the current goal and motivational attitudes. Available findings then do not reflect low-level reflexes but, rather, point to the intimate relationship between emotion and intentional action.

Railton (pp. 326-334) assumes that successful action control relies on a cybernetic loop that initiates actions whenever discrepancies between intended and actual states of affairs are detected. However, efficient control loops do not just wait until discrepancies are detected, they rather make use of predictive models that anticipate probable discrepancies before they actually occur, which in the best case prevents the occurrence of a discrepancy through anticipatory action. Emotion, which has been considered to be the common currency that can signal discrepancies (i.e., the needs for action), might play a crucial role in creating and validating such predictive models. Being the product of such a sophisticated system, emotions are apt to explain action in a way that makes it clear why these actions are rational. The upshot is that rational action does not stand in opposition to, but rather presupposes emotion. 
Moors, Boddez, and De Houwer (2017) also criticize the widespread idea that human action emerges from a competition between action tendencies induced by goal-directed (more rational) processes and action tendencies induced by stimulus-driven emotional (more irrational) processes. According to their assessment, both emotional and nonemotional action tendencies are hardly ever determined by purely stimulus-driven processes, but most often by goal-directed processes. Thus, most of the competition occurs between different goal-directed processes. Both emotional and nonemotional episodes are seen as action control loops in which the degree to which a given stimulus event is related to and discrepant with a current goal is assessed, and the utility of several action (and other) options determines which (and whether an) action tendency will be activated. Emotional and nonemotional action tendencies do not differ in the mechanisms that produce them, but in the degree of control precedence that they have, which ultimately stems from the degree of goal relevance of the events that initiated the loop. Here too, emotional episodes do not need to be seen as separate from action episodes or in opposition to goal-directed action episodes, but rather as special types of goaldirected action episodes.

Finally, Ridderinkhof (2017) argues that the integration of ideomotor theory and Frijda's action-centered approach to emotion (Frijda, 2007) makes for a workable model of emotional action control. He suggests that the processing of stimulus events is informed by current concerns and preferences (a kind of appraisal), which leads to the translation of motives into related action effects that then translate into overt action according to ideomotor principles. Among other things, this approach suggests that emotions contribute to action control by weighing equally suitable action alternatives according to expected costs and benefits (see Hommel, Lippelt, Gurbuz, \& Pfister, in press) and that the experience of emotion does not (necessarily) rely on overt action but on the state of action readiness that a particular event and its appraisal induce. 


\section{Emerging Themes}

While the five contributions differ in terms of emphasis, level of analysis, and theoretical preference, they do show some commonalities and point to a number of shared topics and themes that will provide a useful guideline for further research and theorizing. In particular, we identify at least one source of divergence and three points of convergence.

Theories differ about how they understand the central concepts of emotion and of action, as well as how they see the relation between the two. Older theories, called feelings theories (e.g., James, 1884), take emotion to be synonymous with feelings or emotional experience. Frijda (2007) equates emotion with a special type of action tendency. Some affect program theories (Scarantino, 2017), equate emotion with an affect program. More contemporary theories, called componential theories (e.g., Scherer, 2009), see emotions as episodes comprised of several components, but with no consensus about the exact number and identity of the components to include, and on the relations between these components. A minimal set includes motivation (action tendencies), physiological responses, and feelings. A maximal set adds cognition (information processing) and overt behavior (expressions and fullbody actions). Feeling theories, Scarantino's version of affect program theory, and minimal componential theories see emotion and action as separate phenomena. All three of them, moreover, assume a causal relation between emotion and action. They disagree, however, about the direction of this causal relation: Feeling theories, such as James's (1884) see action as part of the cause of emotion. Scarantino (2017) and minimal componential theories (e.g., Lang, 1994), by contrast, take emotion to be the cause of action. Maximal componential theories (e.g., Moors, 2013), for their part, take action to be part of emotion (i.e., a part-whole relation). Note that the latter theories may still see actions/action tendencies as part of the cause of feelings (as did James, 1884; Moors et al., 2017) or feelings as the cause of actions/action tendencies (as does common sense). Theories 
that see actions/action tendencies as part of emotions, naturally expect processes involved in generating emotions to play a role in action, and vice versa. It is certainly possible that some processes do not overlap, but considering only those when trying to account for emotion and action is unlikely to make for a comprehensive theory.

In addition to the differences in the usage of concepts and the way in which theories understand the relation between emotion and action, the target articles present some striking points of convergence. First, most target articles draw on behavioral and neuroscientific findings to support the Darwinian suggestion that the analysis of stimulus information is not restricted to the coding of physical attributes, such as a snake-like form or the loudness of noise, but also comprises the computation of relational aspects, such as the relevance and implications of the current stimulus event for both current goals and longer term concerns of the perceiver/actor. The consideration of such relational aspects provides important information for action control and for the successful anticipation of action-relevant situational circumstances, thereby making action control more flexible. While that does not rule out the possibility of low-level interactions between less elaborate stimulus representations and primitive motor responses (e.g., LeDoux, 1996), it does suggest that the interacting relational information processes and action processes are strongly interwoven and integrated into a wellfunctioning control machinery in which responsibilities are clearly defined and distributed.

A second point of convergence is that there is increasing dissatisfaction with the common characterization of emotional contributions to action control as (necessarily) irrational and unwanted. Instead, most articles point out that processes involved in emotional episodes provide important information about the goal-relevance of stimulus events (e.g., appraisal) and their implications for ongoing action planning, and both Ridderinkhof (2017) and Moors et al. (2017) consider that many if not all emotional processes do actually operate at the service of instrumental rationality. In some sense, these theoretical approaches can be 
seen as going back to the roots. Neither Darwin (1872) nor James (1884) considered emotions as standing in opposition to effective action control and Darwin strongly emphasized the beneficial evolutionary functions of emotions and their intimate link with action. It is mainly due to the Kantian tradition in philosophy and the strong focus on rational decision-making in psychology and economy that contributions from processes that do not (seem to) follow rational principles were discounted—with Kahneman's (2011) skepticism regarding the rationality of everything nonconscious probably being the most famous example. Recent developments in the cognitive and affective neurosciences have provided strong reasons to doubt this binary view, however. In particular, LeDoux (1996) and Damasio (1994) have emphasized the possibly useful function of emotions for decision-making and action control, especially under uncertainty and time pressure. While their approaches did not take away the irrational flavor of emotions entirely, the present contributions are successful in doing exactly that. While Darwin (1872) saw emotions as functional for our ancestors, the mechanism that he proposed (i.e., serviceable associated habits, which are innate stimulus-response links) is such that individuals from current generations are sometimes pushed into functional behavior and sometimes into nonfunctional behavior, depending on other features of the situation. What the present articles do not yet achieve, however, is to provide theoretical principles and guidelines for how to tell useful and to some degree even rational contributions of emotionrelated processes from truly irrational contributions (at least from the perspective of society) that express themselves in racial biases or religious violence-clearly an item that remains on our to-do list.

A third commonality among the five target articles is that none of them emphasizes the importance of the experiential or feeling dimension of emotion. For one, experience appears to be downplayed in the sense that it is not singled out as requiring special explanation or prediction. As one would expect, this trend is stronger in the psychological and 
neuroscientific articles than in the philosophically minded ones, but even in the latter, phenomenal experience is more used as an argument (as first-person data) than as a phenomenon that needs to be explained in its own right. The explanandum in all of the target articles are action tendencies or action, and the explanans are emotional information processes (be it evaluation of the stimulus or evaluation of action options based on values and expectancies). For another, experience is downplayed in the sense that it is not thought to be necessary in the processing sequence from stimulus to response-a point that is most explicitly subscribed to by Moors et al. (2017). In several target articles, experience is not treated as a full-blown independent variable or a causal mediator, but merely as an epiphenomenon or as a factor that can at best modulate the influence that information processes have on action tendencies and actions. Indeed, nothing about the functions that authors have ascribed to emotional contributions to action control requires agents to have feelings: dangerous stimuli, events violating one's current goals, and response conflict may make one feel bad, but there is no evidence that this emotional experience is an integral part of the underlying computational function. Borrowing from Kirk (1974) it is easy to imagine a well-functioning zombie that is a perfect clone of a human being and fully equipped with an information-processing system, but who lacks the ability to have conscious emotional experience. Still, we may wonder whether it makes much sense to think of these zombies as emotional creatures. Indeed, phenomenal experience does exist and is still the explanandum of Jamesian approaches, in which action is considered as part of the explanans. It is interesting to note that none of the five target articles exploits the available evidence showing that action can systematically affect the experience of emotion and value judgements made on the basis of emotional experience (Niedenthal et al., 2005). This raises the question of how the two approaches (action vs. experience as explanandum) and the respective supportive evidence relate to each other - a second item on our to-do list. 


\section{Towards Integration}

In an attempt to advance the integration of emotion and action somewhat further, let us take a step back, and pursue the idea that emotion and action are dependent variables, that is, phenomena that scientists need to explain and predict, without presupposing that they interact causally. For instance, they may be partly identical in terms of their underlying processes and substrates. The same argument has been made for the concepts of perception and action, which have been taken to refer to the exact same processes but merely emphasize different functions (i.e., providing information about vs. changing the actor-environment relationship; see Hommel, Müsseler, Aschersleben, \& Prinz, 2001). This still left the task to identify the functional and neural processes that eventually generate a given perceptual experience or an overt action, but it does allow for many of these processes to overlap and to render it unreasonable to consider some of these processes "perceptual" and others "actional." Along these lines, it may be misleading to label some processes involved in generating emotions "emotional" or "affective" and others "action-related" or "cognitive" - as this would imply that their exclusive purpose consists in either generating an emotion or controlling an action.

The hypothesis that emotion and action are partly identical in terms of the underlying processes and substrate is likely to facilitate the integration of seemingly disparate or competing approaches. Partial identity of emotion and action allows for the impact of emotionally relevant stimuli (not "perception" or "emotion") on action control一 the research topic emphasized by the present contributions - just as well as for the impact of facial or body movement (not "action") on emotional experience. This is because the processing of emotional stimuli feeds into both emotional experience and action control while body movements do not only require action control but also produce reafferent information that feeds into emotional experience. In the light of this partial identity, we may also do away with 
the conceptualization of all the processes feeding into emotional experience as irrational, simply because many of them will also feed into the control of rational, goal-directed action.

\section{References}

Blakemore, R. L., \& Vuilleumier, P. (2017). An emotional call to action: Toward an integrative motor-affective neuroscience model. Emotion Review, 9(4), 299-309.

Damasio, A. R. (1994). Descartes' error: Emotion, reason and the human brain. New York, NY: Avon. Darwin, C. R. (1872). The expression of the emotions in man and animals. London, UK: John Murray.

Frijda, N. H. (2007). The laws of emotion. Mahwah, NJ: Erlbaum.

Hommel, B., Lippelt, D. P., Gurbuz, E., \& Pfister, R. (in press). Contributions of expected sensory and affective action effects to action selection and performance: Evidence from forced- and free-choice tasks. Psychonomic Bulletin \& Review.

Hommel, B., Müsseler, J., Aschersleben, G., \& Prinz, W. (2001). The theory of event coding (TEC): A framework for perception and action planning. Behavioral and Brain Sciences, 24, 849-878.

James, W. (1884). What is an emotion? Mind, 9, 188-205.

Kahneman, D. (2011). Thinking, fast and slow. New York, NY: Farrar, Straus \& Giroux. Kirk, R. (1974). Sentience and behavior. Mind, 83, 43-60.

Lang, P. J. (1994). The varieties of emotional experience: A meditation on James-Lange theory. Psychological Review, 101, 211-221.

LeDoux, J. E. (1996). The emotional brain. New York, NY: Simon \& Schuster. Moors, A. (2013). On the causal role of appraisal in emotion. Emotion Review, 5, 132-140. Moors, A., Boddez, Y., \& De Houwer, J. (2017). The power of goal-directed processes in the causation of goal-directed and other actions. Emotion Review, 9(4), 310-318. 
Niedenthal, P. M., Barsalou, L., Winkielman, P., Krauth-Gruber, S., \& Ric, F. (2005).

Embodiment in attitudes, social perception, and emotion. Personality and Social Psychology Review, 9, 184-211.

Railton, P. (2017). At the core of our capacity to act for a reason: The affective system and evaluative model-based learning and control. Emotion Review, 9(4), 335-342.

Ridderinkhof, K. R. (2017). Emotion in action: A predictive processing perspective and theoretical synthesis. Emotion Review, 9(4), 319-325.

Scarantino, A. (2017). Do emotions cause actions and if so, how? Emotion Review, 9(4), 326334.

Scherer, K. R. (2009). The dynamic architecture of emotion: Evidence for the component process model. Cognition and Emotion, 23, 1307-1351.

Susskind, J. M., Lee, D. H., Cusi, A., Feiman, R., Grabski, W., \& Anderson, A. K. (2008). Expressing fear enhances sensory acquisition. Nature Neuroscience, 11, 843-850. 